\title{
Metronidazole Induced Neurotoxicity in a Case of Liver Abscess- A Case Report
}

\author{
Sourabh Mishra ${ }^{1}$, Saurabh Goel ${ }^{2}$, Madhubala Gaur ${ }^{3}$ \\ ${ }^{1}$ Postgraduate Resident, ${ }^{2}$ H.O.D \& Professor, ${ }^{3}$ Professor, Gen Surgery Dept, \\ Saraswathi Institute of Medical Sciences, Hapur
}

\begin{abstract}
Metronidazole is a commonly used drug and considered relatively safe. But it may present with neurotoxicity, commonly peripheral and rarely central. We report here a case of a young patient with amoebic liver abscess who continued taking metronidazole longer than the prescribed duration of the drug and developed peripheral neuropathy and cerebellar neurotoxicity which was reversible after discontinuation of the drug.
\end{abstract}

Keywords: Ataxia, Liver Abscess, Metronidazole, Neurotoxicity

\section{Introduction}

Metronidazole is a nitroimidazole derivative. It has broad spectrum cidal activity against protozoa and most anaerobic bacteria. It is a commonly prescribed drug for amoebiasis, giardiasis, trichomoniasis and anaerobic bacterial infections such as brain abscess and puerperal sepsis. Its use is considered relatively safe. The most frequently observed adverse effects of metronidazole is nausea, vomiting, metallic taste and epigastric distress. Neurotoxicity is less commonly seen. Central neurotoxicity is rare as compared to peripheral neuropathy and may necessitate its withdrawal. The exact incidence of metronidazole induced encephalopathy is not known but usually occurs after a prolonged cumulative dose. MRI findings along with clinical features help in reaching the diagnosis. We present a case of a young male with liver abscess presenting with metronidazole induced neurotoxicity.

\section{Case Report}

A 30-year-old male presented to our hospital in the first week of march 2019 with slurring of speech, vertigo, instability in walking and burning sensation

\section{Corresponding author:}

\section{Sourabh Mishra,}

Postgraduate, Gen Surgery Dept, Saraswathi institute of Medical Sciences,

Email: sammishra333@gmail.com, over feet and hands below wrist for two days. There was no history of fever, convulsions, trauma/fall, headache, vomiting or diarrhea.

Patient gave history of admission in our hospital in the month of January 2019, six weeks prior to the present visit with chief complaints of high grade fever associated with chills, pain in right hypochondrium and decreased appetite for five days. On examination he was febrile with temperature of $103 \mathrm{f}$, his pulse and blood pressure was $112 / \mathrm{min}$ and $120 / 76 \mathrm{mmHg}$ respectively. There was no icterus or any lymphadenopathy on general physical examination. Systemic examination revealed tender hepatomegaly.

Patient was investigated and found to have an abscess in posterior segment of right lobe of liver with volume of $300 \mathrm{cc}$. Based on biochemical tests and ultrasonography of abdomen, a diagnosis of amoebic liver abscess was made. Patient was treated with parenteral metronidazole $800 \mathrm{mg}$ eight hourly for ten days. The patient improved clinically and was discharged on oral metronidazole $800 \mathrm{mg}$ thrice a day for a week. But patient continued metronidazole for another five weeks. After six weeks of discharge, patient presented with slurring of speech, vertigo, instability in walking and burning sensation in the feet and hands for two days. On examination the patient had staccato speech, dysmetria, ataxic gait, dysdiadochokinesia, past-pointing suggestive of cerebellar involvement. 
Serial routine blood investigations were normal including complete blood count, liver function test, kidney function test. Vitamin B12 and fasting blood sugar levels were normal. Amoebic serology was positive. Ultrasonography of the abdomen showed hepatomegaly with liver abscess of $258 \mathrm{cc}$ with an area of liquefaction noted in posterior segment of right lobe of liver abutting the diaphragm. Nerve conduction studies of both lower limbs revealed motor sensory neuropathy.

Non contrast computed tomography (NCCT) scan of brain was normal. Magnetic resonance imaging (MRI) scan of brain showed areas of altered signal intensity in bilateral dentate nuclei of cerebellar hemispheres and deep white matter of right parietal region which appeared hyperintense on $\mathrm{T} 2 \mathrm{~W}$ images. There were also multiple foci of blooming on SWI images in bilateral cerebellar hemispheres suggestive of microhemorrhages. Computed tomography angiography ruled out any vascular lesion. Repeat MRI scan done three months later showed resolution of the previous lesions.

\section{Discussion}

Metronidazoleiscommonlyusedantibioticforvarious anaerobic and protozoan infections ${ }^{(1)}$. It is considered a relatively safe drug, but can cause neurotoxicity such as ataxia, seizure, peripheral neuropathies, cerebellar signs and symptoms and encephalopathy ${ }^{(2)}$. The true incidence of metronidazole induced neurotoxicity is not known. There are several case reports of metronidazole induced peripheral neuropathy but we could find only few reported cases of acute neurotoxicity and cerebellar dysfunction with associated MRI findings ${ }^{(3-5)}$. cerebellar toxicity is an unusual manifestation of metronidazole toxicity. The cumulative dose of metronidazole leading to neurotoxicity ranges from 25-110grms.

The patient presented with acute cerebellar toxicity and peripheral sensory motor neuropathy after consuming a cumulative dose of around 75 grams. The signs of cerebellar toxicity improved within 3-4 days of stopping metronidazole but symptoms of neuropathy showed some improved only after 20-25 days.

Magnetic resonance imaging brain showed areas of hyperintense lesions on T2-weight/FLAIR images in right parietal region and bilateral dentate nuclei of cerebellar hemispheres. Computed tomography angiography was done which ruled out any vascular lesion. Repeat MRI brain done three months later was normal.

Improvement in symptoms on discontinuation of metronidazole, bilateral symmetrical diffuse lesions, normal computed tomography angiography and resolution of findings in repeat MRI favoured the diagnosis of metronidazole induced reversible neurotoxicity.

The exact mechanism of metronidazole induced neurotoxicity is not known. Ahmed et al. speculated the lesions to be due to axonal swelling with increased water content because of reversibility of lesions ${ }^{(6)}$. Another mechanism includes possibility of vascular spasms producing mild reversible localised ischemic changes. Modulation of gamma amino butyric acid (GABA) receptors within the cerebellar and vestibular systems has also been proposed. Binding of metabolites of metronidazole to ribonucleic acid (RNA) instead of deoxyribonucleic acid (DNA). Inhibiting RNA protein synthesis leading to axonal degeneration has also been suggested.

Ahmed et al. first described the imaging findings of metronidazole neurotoxicity in $1995^{(6)}$. Since then 17 such cases have been reported. Typical MRI findings include T2 hyperintense lesions in cerebellar dentate nuclei, tectum, red nucleus and tegmentum around periaqueductal Gray matter, dorsal pons and medulla and corpus callosum ${ }^{(7,8)}$. White matter of cerebrum is involved uncommonly. The differential diagnosis of bilateral symmetric T2 hyperintense lesions in dentate nuclei is methyl bromide intoxication, maple syrup urine disease and enteroviral encephalomyelitis ${ }^{(9)}$. This case is unique as in review of literature we could not find a case of metronidazole neurotoxicity with multiple microhemorrhages in bilateral cerebral and cerebellar hemispheres.

The diagnosis of metronidazole encephalopathy is made by MRI findings in conjunction with clinical findings and treatment history of patient. With cessation of metronidazole treatment, the imaging findings and clinical symptoms resolve ${ }^{(10)}$. Review of literature showed symptoms of encephalopathy to improve within days of stopping metronidazole while neuropathy may persist for months ${ }^{(5)}$. Similar were the observation in our 
patient.

\section{Conclusion}

Since surgeons treat a large number of patients with metronidazole, they should be aware of doses and duration of therapy and vigilant about the possible neurotoxicity.

Ethical Clearance- Taken from Saraswathi institute of medical sciences institutional ethical committee.

\section{Source of Funding- Self}

\section{Conflict of Interest- Nil}

\section{References}

1. Bennett JE, Dolin R, Blaser MJ. Mandell, Douglas and Bennetts Principles and Practice of Infectious Diseases, 8ed. Philadelphia: Elsevier Health sciences; 2014. p. 2753.

2. Gupta AK, Agarwal MP, Avasthi R, Bhadoria DP, Rohatgi N. Metronidazole-induced neurotoxicity. J Assoc physicians India 2003 Jun;51:617-8.

3. Lawford R, Sorrell TC. Amebic abscess of spleen complicated by metronidazole-induced neurotoxicity: case report. Clin Infect Dis 1994 Aug;19(2):346-8.

4. Ito H, Maruyama M, Ogura N, et al. Reversible cerebellar lesions induced by metronidazole therapy for helicobacter pylori. J Neuroimaging 2004 Oct;14(4):369-71.

5. Iqbal A, Tripathi K, Rai M, Dwivedi AN. Clinical and imaging perspective and unanswered questions in a case of metronidazole induced encephalopathy. Ann Indian Acad Neurol 2013 Oct;16(4):569-71.

6. Ahmed A, Loes DJ, Bressler EL. Reversible magnetic resonance imaging findings in metronidazole-induced encephalopathy. Neurology 1995 Mar;45(3 Pt 1):588-9.

7. Woodruff BK, Wijdicks EF, Marshall WF. Reversible metronidazole induced lesions of the cerebellar dentate nuclei. N Engl J Med 2002 Jan 3;346(1):68-9.

8. Bonkowsky JL, Sondrup C, Benedict SL. Acute reversible cerebellar lesions associated with metronidazole therapy. Neurology 2007 Jan 16;68(3):180.

9. Kalia V, Vibhuti, Saggar K. Case report: MRI of the brain in metronidazole toxicity. Indian J Radiol Imaging 2010 Aug;20(3):195-7.

10. Chandak S, Agarwal A, Shukla A, Joon P. A Case Report of metronidazole induced neurotoxicity in Liver Abscess patient and the Usefulness of MRI for its Diagnosis. J Clin Diagn Res 2016 Jan;10(1):TDo6-7. 